PROCEEDINGS OF THE

AMERICAN MATHEMATICAL SOCIETY

Volume 119, Number 4, December 1993

\title{
UNITARY $\mathbb{Z}^{d}$-ACTIONS WITH CONTINUOUS SPECTRUM
}

\author{
VITALY BERGELSON, ISAAC KORNFELD, AND BORIS MITYAGIN
}

(Communicated by Palle E. T. Jorgensen)

\begin{abstract}
For any unitary $\mathbb{Z}^{d}$-action on a Hilbert space with continuous spectrum weakly wandering vectors are dense. This wandering can be forced to occur along $I P$-sets. This is a generalization and strengthening of a result due to Krengel. Our method is based on the contractive mapping fixed-point theorem.
\end{abstract}

Among other results Krengel obtained in $[\mathrm{K}]$ an interesting characterization of unitary operators with continuous spectrum. Let $U$ be a unitary operator acting on a Hilbert space $H$. A vector $f \in H$ is called weakly wandering with respect to $U$ if there exists a doubly infinite strictly increasing sequence $\left\{n_{i}\right\}_{i \in \mathbb{Z}}$ such that $\left\langle U^{n_{i}} f, U^{n_{j}} f\right\rangle=0$ for all distinct $i, j$. Krengel proved that $U$ has continuous spectrum (i.e., $U$ has no eigenvectors) if and only if the weakly wandering vectors are dense in $H$. As a matter of fact, the proof in $[\mathrm{K}]$ is given for isometries; in that case the wandering occurs along one-sided increasing sequences. However, essentially the same proof when applied to unitary operators yields wandering along a double infinite sequence. Krengel's original proof is quite intricate and does not seem to be easily susceptible to generalization and refinement.

The purpose of this note is to suggest a new and quite simple approach which nonetheless allows us to strengthen Krengel's result in the following two directions.

First, we show that the result holds for unitary $\mathbb{Z}^{d}$-actions. The method actually works for a wide class of groups (see the concluding remarks in $\S 4$ ). We confine ourselves to $\mathbb{Z}^{d}$-actions to keep this note short and to make clear the core of our approach. A Krengel-type theorem for $d$-parametric groups was also proved by Graham [G]. However, the proof in [G] is even more complicated than that given in $[\mathrm{K}]$ and does not seem to be generalizable to, say, noncompactly generated abelian groups.

Second, we show that sequences along which wandering occurs may be guaranteed to have an additional arithmetic structure, namely, that of an $I P$-set.

Received by the editors January 21, 1992.

1991 Mathematics Subject Classification. Primary 47B99; Secondary 28D05. 
Definition 1. Given an infinite set $A \subset \mathbb{Z}^{d}$, the IP-set $\Gamma$ generated by $A$ is the set

$$
\Gamma=\left\{x_{1}+\cdots+x_{k} \mid x_{i} \in A, x_{i} \neq x_{j} \text { for } i \neq j, \quad 1 \leq i, j \leq k, k \in \mathbb{N}\right\}
$$

of all finite sums of distinct elements from $A$. We shall often use the notation $\Gamma=F S(A)$. An $I P$-set $\Gamma$ is called symmetric if $\Gamma=F S(B)$, where $B$ is a symmetric subset in $\mathbb{Z}^{d}$ (i.e., $x \in B \Rightarrow-x \in B$ ).

$I P$-sets may be viewed as generalized subsemigroups of $\mathbb{Z}^{d}$. The notion of $I P$-set has proved useful in many situations in ergodic theory and topological dynamics, where it turns out to be helpful to study iterations of operators along IP-sequences (cf. [F]).

Definition 2. A family of operators $\left\{U_{n}\right\}_{n \in \mathbb{Z}^{d}}$ on a Hilbert space $H$ is called a $\mathbb{Z}^{d}$-action if $U_{n+m}=U_{n} U_{m} \quad \forall n, m \in \mathbb{Z}^{d}$. Such an action is called unitary if all $U_{n}, n \in \mathbb{Z}^{d}$, are unitary operators.

A nonzero vector $f \in H$ is called an eigenvector for the unitary $\mathbb{Z}^{d}$-action $\left\{U_{n}\right\}_{n \in \mathbb{Z}^{d}}$ on $H$ if there exist complex numbers $\lambda_{n}, n \in \mathbb{Z}^{d}$, such that $U_{n} f=$ $\lambda_{n} f \quad \forall n \in \mathbb{Z}^{d}$.

A unitary $\mathbb{Z}^{d}$-action is said to have continuous spectrum if it has no eigenvectors.

Main Theorem. A unitary $\mathbb{Z}^{d}$-action $\left\{U_{n}\right\}_{n \in \mathbb{Z}^{d}}$ on a Hilbert space $H$ has continuous spectrum if and only if for any $f \in H$ and any $\varepsilon>0$ there exits $\tilde{f}$, $\|f-\tilde{f}\|<\varepsilon$, and a symmetric IP-set $\Gamma \subset \mathbb{Z}^{d}$ such that

$$
\left\langle U_{\alpha} \tilde{f}, U_{\beta} \hat{f}\right\rangle=0 \quad \text { for all distinct } \alpha, \beta \in \Gamma .
$$

In the next section we collect a few auxiliary facts. A proof of the main theorem is given in $\S 3$. Section 4 is devoted to further discussion and some concluding remarks.

\section{2}

The following Proposition is well known for $\mathbb{Z}$-actions; it goes back to Koopman and von Neumann $[\mathrm{KN}]$. The extension of $\mathbb{Z}^{d}$-actions is routine.

We recall that a set $S \subset \mathbb{Z}^{m}$ is said to have density 1 if

$$
d(S)=\lim _{N \rightarrow \infty} \frac{\#\left(S \cap[-N, N]^{m}\right)}{(2 N+1)^{m}}=1 .
$$

Proposition. For a unitary $\mathbb{Z}^{m}$-action $\left\{U_{n}\right\}_{n \in \mathbb{Z}^{m}}$ on a Hilbert space $H$, the following statements are equivalent:

(i) The action $\left\{U_{n}\right\}_{n \in \mathbb{Z}^{m}}$ has continuous spectrum.

(ii) For any $f, g \in H$ there exists a set $S \subset \mathbb{Z}^{m}$ with $d(S)=1$ such that

$$
\lim _{\substack{n \rightarrow \infty \\ n \in S}}\left\langle U_{n} f, g\right\rangle=0 \text {. }
$$

(iii) For all $f, g \in H$

$$
\lim _{N \rightarrow \infty} \frac{1}{(2 N+1)^{m}} \sum_{n \in[-N, N]^{m}}\left|\left\langle U_{n} f, g\right\rangle\right|=0 .
$$


(iv) If for $f \in H$ the orbit $\left\{U_{n} f\right\}_{n \in \mathbb{Z}^{d}}$ is precompact in the strong topology, then $f=0$.

We use the following notation. For an $I P$-set $\Gamma \subset \mathbb{Z}^{d}$, put $\Gamma^{\prime}:=\Gamma \backslash\{0\}$. For $S \subset \mathbb{Z}^{d}$ and $c \in \mathbb{Z}, c \neq 0$, put $S / c:=\left\{n \in \mathbb{Z}^{d} \mid c n \in S\right\}$. For $S \subset \mathbb{Z}^{d}$ and $n \in \mathbb{Z}^{d}$, put $S-n:=\left\{l \in \mathbb{Z}^{d} \mid l+n \in S\right\}$. For $S \subset \mathbb{Z}^{d}$ and $c \in \mathbb{N}$, put

$$
c S:=\left\{s_{1}+s_{2}+\cdots+s_{k} \mid s_{i} \in S, 1 \leq i \leq k, 1 \leq k \leq c\right\} .
$$

In other words $c S$ is the set of all $k$-element sums formed by elements from $S$ for $k=1,2, \ldots, c$.

Lemma 1. Let the set $S \subset \mathbb{Z}^{d}$ have density 1 . Then $d(S / c)=1$ for any $c \in \mathbb{Z}$, $c \neq 0$, and $d(S-n)=1$ for any $n \in \mathbb{Z}^{d}$. If the sets $S_{1}, \ldots, S_{k}$ have density 1 , then $D\left(\bigcap_{1 \leq i \leq k} S_{i}\right)=1$.

Proof. The proof is straightforward.

Lemma 2. Let $S \subset \mathbb{Z}^{d}$ have density 1 . For any $c \in \mathbb{N}$ there exists a symmetric IP-set $\Gamma=F S\left(\left\{ \pm n_{i}\right\}_{i=1}^{\infty}\right)$ such that $(c \Gamma)^{\prime} \subset S$.

Proof. By Lemma 1 the set $S_{1}=\bigcap_{0<|i| \leq c} S / i$ has density 1 and, in particular, is nonempty. Pick $n_{1} \in S_{1}$. Then $i n_{1} \in S$ for any $i, 0<|i| \leq c$. Define $S_{2}=\bigcap_{0 \leq|i| \leq c, 0<|j| \leq c}\left(S-i n_{1}\right) / j$. By Lemma 1 there exists $n_{2} \in S_{2}$. Then

$$
i n_{1}+j n_{2} \in S \cup\{0\} \quad \forall i, j,-c \leq i, j \leq c .
$$

Assume that $n_{1}, n_{2}, \ldots, n_{k}$ have been already picked in such a way that $\sum_{j=1}^{k} i_{j} n_{j} \subset S \cup\{0\}$ for all $\left\{i_{j}\right\}_{1}^{k},\left|i_{j}\right| \leq c$. Let

$$
S_{k+1}=\bigcap\left(S-\sum_{j=1}^{k} i_{j} n_{j}\right) / i_{k+1},
$$

where the intersection is taken over all $i_{j}, 1 \leq j \leq k+1$, such that $-c \leq$ $i_{1}, \ldots, i_{k} \leq c$ and $0<\left|i_{k+1}\right| \leq c$.

By Lemma 1, $S_{k+1}$ has density 1. Pick $n_{k+1} \in S_{k+1}$. Then

$$
\sum_{j=1}^{k+1} i_{j} n_{j} \subset S \cup\{0\} \quad \forall i_{j}, 0 \leq j \leq k+1 \text { with } 0 \leq\left|i_{j}\right| \leq c .
$$

This process gives us a sequence $\left\{n_{i}\right\}_{i=1}^{\infty}$ such that $c \Gamma \subset S \cup\{0\}$ for $\Gamma=$ $F S\left(\left\{ \pm n_{i}\right\}_{i=1}^{\infty}\right)$.

Lemma 3. Let $\left\{U_{n}\right\}_{n \in \mathbb{Z}^{d}}$ be a unitary $\mathbb{Z}^{d}$-action on a Hilbert space $H$ with continuous spectrum. Let $f \in H, c \in \mathbb{N}$. Then for any $\eta>0$ there exists a symmetric IP-set $\Gamma=F S\left(\left\{ \pm n_{i}\right\}_{i=1}^{\infty}\right)$ such that $\sum_{n \in(c \Gamma)^{\prime}}\left|\left\langle U_{n} f, f\right\rangle\right|<\eta$.

Proof. By the Proposition the set $\left\{n \in \mathbb{Z}^{d}\left|\left\langle U_{n} f, f\right\rangle\right|\langle\theta\}\right.$ has density 1 for any $\theta>0$. Then by Lemma 1 , for any $\theta>0$ and any finite set $F \subset H$, the set

$$
A_{F, \theta}=\left\{n \in \mathbb{Z}^{d}\left|\sum_{0<|i| \leq c} \sum_{g \in F}\right|\left\langle U_{i n} g, f\right\rangle \mid<\theta\right\}
$$


also has density 1 . Let $n_{1} \in A_{F_{1}, \eta / 2}$, where $F_{1}$ is the singleton $\{f\}$. If for $j \geq 1$ the points $n_{1}, \ldots, n_{j}$ have already been picked, put $\Gamma_{j}=F S\left\{ \pm n_{i}\right\}_{i=1}^{j}$, then take $F_{j}=\left\{U_{n} f \mid n \in \Gamma_{j}\right\}$, and pick $n_{j+1} \in A_{F_{j}}, \eta / 2^{j}$. Continuing in this way we get an infinite sequence $\left\{n_{i}\right\}_{i=1}^{\infty}$ such that $\Gamma=F S\left(\left\{ \pm n_{i}\right\}_{i=1}^{\infty}\right)$ gives us the desired symmetric $I P$-set.

Proof of Main Theorem. We give the "only if" part only. The "if" part follows easily from (iv) of the proposition in $\S 2$.

Without loss of generality we may assume that $\langle f, f\rangle=1$.

Let $\varepsilon, 0<\varepsilon<1$, be given. Put $c=6$ and $\eta=\varepsilon / 4$, and by Lemma 3 choose a symmetric $I P$-set

$$
\Gamma=F S\left(\left\{ \pm n_{i}\right\}_{i=1}^{\infty}\right) \quad \text { such that } \sum_{n \in\left(6 \Gamma^{\prime}\right)}\left|\left\langle U_{n} f, f\right\rangle\right|<\varepsilon / 4 .
$$

We shall show that there exist complex numbers $h_{\alpha}$,

$$
h_{-\alpha}=\overline{h_{\alpha}}, \quad \alpha \in E=\Gamma-\Gamma,
$$

such that

$$
\sum_{\alpha \in E}\left|h_{\alpha}\right|<\varepsilon
$$

and

$$
\left\langle U_{n} \tilde{f}, U_{m} \tilde{f}\right\rangle=0 \quad \text { for any distinct } n, m \in \Gamma,
$$

where

$$
\tilde{f}=f+\sum_{\alpha \in E} h_{\alpha} U_{\alpha} f .
$$

Inequality $\left(1^{\prime}\right)$ implies that $\|f-\tilde{f}\|<\varepsilon$ and $\|\tilde{f}\|>1-\varepsilon>0$, but we require more, namely,

$$
\langle\tilde{f}, \tilde{f}\rangle=1 .
$$

If $\tilde{f}$ satisfies (3), then conditions (1), (2), (4) are equivalent to the infinite system of nonlinear (quadratic) equations in the indeterminates $h_{\alpha}, \alpha \in E$. Indeed, for $n, m \in \Gamma$,

$$
\begin{aligned}
\left\langle U_{n} \tilde{f}, U_{m} \tilde{f}\right\rangle= & \left\langle U_{n}\left(f+\sum_{\alpha \in E} h_{\alpha} U_{\alpha} f\right), U_{m}\left(f+\sum_{\beta \in E} h_{\beta} U_{\beta} f\right)\right\rangle \\
= & \left\langle U_{n-m} f, f\right\rangle+\sum_{\alpha \in E} h_{\alpha}\left\langle U_{n-m+\alpha} f, f\right\rangle+\sum_{\beta \in E} \bar{h}_{\beta}\left\langle U_{n-m-\beta} f, f\right\rangle \\
& +\sum_{\alpha, \beta \in E} h_{\alpha} \bar{h}_{\beta}\left\langle U_{n-m+\alpha-\beta} f, f\right\rangle .
\end{aligned}
$$

Put

$$
b_{\lambda}^{\prime}= \begin{cases}-\frac{1}{2} b_{\lambda}, & \lambda \neq 0 \\ 0, & \lambda=0\end{cases}
$$


where $b_{\lambda}=\left\langle U_{\lambda} f, f\right\rangle, \lambda \in \mathbb{Z}^{d}$. (Notice that $\left|b_{\lambda}\right| \leq 1$ and $b_{-\lambda}=\overline{b_{\lambda}}, \lambda \in \mathbb{Z}^{d}$.) With this notation conditions $(2),(4)$ become the system of equations

$$
\sum_{\alpha \in E} h_{\alpha} b_{\lambda+\alpha}+\frac{1}{2} \sum_{\alpha, \beta \in E} h_{\alpha} \bar{h}_{\beta} b_{\lambda+\alpha-\beta}=b_{\lambda}^{\prime}, \quad \lambda \in E .
$$

Notice that condition (4) corresponds to the case $\lambda=0$ in (5).

Now consider the space

$$
l_{\text {symm }}^{1}(E)=\left\{x=\left(x_{\alpha}\right)_{\alpha \in E}, \quad x_{-\alpha}=\bar{x}_{\alpha}\left|\|x\|=\sum_{\alpha \in E}\right| x_{\alpha} \mid<\infty\right\}
$$

of symmetric summable functions (sequences) on $E$.

The function $b_{\lambda}^{\prime}, \lambda \in E$, is bounded (by 1/2) on $E$ and symmetric; it follows that the operator $\Phi: x=\left(x_{\alpha}\right) \mapsto y=\left(y_{\lambda}\right)$, where

$$
y_{-\lambda}=b_{\lambda}^{\prime}-\sum_{\substack{\alpha \in E \\ \alpha \neq-\lambda}} b_{\lambda+\alpha} x_{\alpha}-\frac{1}{2} \sum_{\alpha, \beta \in E} b_{\lambda+\alpha-\beta} x_{\alpha} \bar{x}_{\beta}
$$

is well defined as an operator from $l^{1}(E)$ into $l^{\infty}(E)$ and the image $y=\Phi x$ is a symmetric function if $x \in l_{\text {symm }}^{1}(E)$.

We shall show that:

(i) $\Phi$ maps $l_{\text {symm }}^{1}(E)$ into itself.

(ii) The ball $B_{\tau}=\left\{x \in l_{\text {symm }}^{1}(E):\|x\| \leq \tau\right\}$ is invariant under $\Phi$ if $\frac{\varepsilon}{5} \leq \tau \leq$ $\varepsilon$.

(iii) $\Phi$ is contractive in $B_{\tau}$ if $\tau<\left(1-\frac{\varepsilon}{4}\right) /\left(1+\frac{\varepsilon}{4}\right)$; more precisely,

$$
\left\|\Phi(x)-\Phi\left(x^{\prime}\right)\right\| \leq \theta_{1}\left\|x-x^{\prime}\right\|, \quad x, x^{\prime} \in B_{\tau},
$$

where $\theta_{1}=\theta+\frac{\varepsilon}{4}(1-\theta), \theta:=\tau\left(1+\frac{\varepsilon}{4}\right) /\left(1-\frac{\varepsilon}{4}\right)<1$.

To prove (i) let us estimate $\|\Phi(x)\|$. By (6) we have

$$
\|\Phi(x)\|=\sum_{\lambda \in E}\left|y_{\lambda}\right| \leq\left\|\Sigma_{0}\right\|+\left\|\Sigma_{1}\right\|+\left\|\Sigma_{2}\right\|
$$

where $\Sigma_{0}, \Sigma_{1}, \Sigma_{2}$ are constant, linear, and quadratic parts of $\left\{y_{\lambda}\right\}$ respectively. Recall that $E=\Gamma-\Gamma, 0 \in \Gamma$, and $\Gamma$ is symmetric; therefore, $E=\Gamma+\Gamma=2 \Gamma \subset 2 E \subset 3 E \subset 6 \Gamma$. Then

$$
\begin{aligned}
\left\|\Sigma_{1}\right\| & =\sum_{\lambda \in E}\left|\sum_{\substack{\alpha \in E \\
\alpha \neq-\lambda}} b_{\lambda+\alpha} x_{\alpha}\right| \leq \sum_{\alpha \in E}\left|x_{\alpha}\right| \sum_{\substack{\lambda \in E \\
\lambda \neq-\alpha}}\left|b_{\lambda+\alpha}\right| \\
& \left.\leq \sum_{\alpha \in E}\left|x_{\alpha}\right|\left(\sum_{\lambda \in(2 E)^{\prime}}\left|b_{\lambda}\right|\right)\right) \leq \frac{\varepsilon}{4}\|x\|
\end{aligned}
$$

and

$$
\begin{aligned}
\left\|\Sigma_{2}\right\| & =\frac{1}{2} \sum_{\lambda \in E}\left|\sum_{\alpha, \beta \in E} b_{\lambda+\alpha-\beta} x_{\alpha} \overline{x_{\beta}}\right| \leq \frac{1}{2} \sum_{\alpha, \beta \in E}\left|x_{\alpha}\right|\left|\bar{x}_{\beta}\right|\left(1+\sum_{\substack{\lambda \in E \\
\lambda \neq \beta-\alpha}}\left|b_{\lambda+\alpha+\beta}\right|\right) \\
& \leq \frac{1}{2} \sum_{\alpha, \beta \in E}\left|x_{\alpha}\right|\left|x_{\beta}\right|\left(1+\sum_{\lambda \in(3 E)^{\prime}}\left|b_{\lambda}\right|\right) \leq \frac{1}{2}\left(1+\frac{\varepsilon}{4}\right)\|x\|^{2} .
\end{aligned}
$$


Of course,

$$
\left\|\Sigma_{0}\right\| \leq \frac{1}{2} \sum_{\lambda \in E^{\prime}}\left|b_{\lambda}\right| \leq \frac{\varepsilon}{8}
$$

Therefore,

$$
\|\Phi(x)\| \leq \frac{\varepsilon}{8}+\frac{\varepsilon}{4}\|x\|_{1}+\frac{1}{2}\left(1+\frac{\varepsilon}{4}\right)\|x\|^{2} \quad \forall x \in l_{\text {symm }}^{1}(E),
$$

and $\Phi(x)$ is symmetric; this proves (i).

If $x \in B_{\tau}$, i.e., $\|x\| \leq \tau$, then

$$
\begin{aligned}
\|\Phi(x)\| & \leq \frac{\varepsilon}{8}+\frac{\varepsilon}{4} \tau+\frac{1}{2}\left(1+\frac{\varepsilon}{4}\right) \tau^{2} \\
& =\frac{\varepsilon}{8}(1+\tau)^{2}+\frac{1}{2} \tau^{2} \leq \tau \quad \text { for } \frac{\varepsilon}{5} \leq \tau \leq \varepsilon .
\end{aligned}
$$

This proves (ii).

To prove (iii) take $x=\left\{x_{\alpha}\right\}, x^{\prime}=\left\{x_{\alpha}^{\prime}\right\}$ in $B_{\tau}$, and let $d=\left\|x-x^{\prime}\right\|$.

We have, similarly to (7),

$$
\left\|\Phi(x)-\Phi\left(x^{\prime}\right)\right\| \leq\left\|\sigma_{1}\right\|+\left\|\sigma_{2}\right\|,
$$

where $\sigma_{1}$ and $\sigma_{2}$ correspond respectively to linear and quadratic terms. Notice that the constant terms are the same in $\Phi(x)$ and $\Phi\left(x^{\prime}\right)$,

$$
\begin{aligned}
\left\|\sigma_{1}\right\| \leq & \sum_{\lambda \in \Gamma} \sum_{\substack{\alpha \in E \\
\alpha \neq-\lambda}}\left|b_{\lambda+\alpha}\right|\left|x_{\alpha}-x_{\alpha}^{\prime}\right|=\sum_{\alpha \in E}\left|x_{\alpha}-x_{\alpha}^{\prime}\right|\left(\sum_{\substack{\lambda \in E \\
\lambda \neq-\alpha}}\left|b_{\lambda+\alpha}\right|\right) \leq \frac{\varepsilon}{4} d ; \\
\left\|\sigma_{2}\right\| \leq & \frac{1}{2} \sum_{\lambda \in E} \sum_{\alpha, \beta \in E}\left|b_{\lambda+\alpha-\beta}\right|\left|x_{\alpha} \bar{x}_{\beta}-x_{\alpha}^{\prime} \bar{x}_{\beta}^{\prime}\right| \\
\leq & \frac{1}{2} \sum_{\lambda, \alpha, \beta \in E}\left|b_{\lambda+\alpha-\beta}\right|\left\{\left|x_{\alpha} \bar{x}_{\beta}-x_{\alpha} \bar{x}_{\beta}^{\prime}\right|+\left|x_{\alpha} \bar{x}_{\beta}^{\prime}-x_{\alpha}^{\prime} \bar{x}_{\beta}^{\prime}\right|\right\} \\
\leq & \frac{1}{2} \sum_{\alpha, \beta \in E}\left|x_{\alpha}\right|\left|x_{\beta}-x_{\beta}^{\prime}\right| \cdot \sum_{\lambda \in E}\left|b_{\lambda+\alpha-\beta}\right| \\
& +\frac{1}{2} \sum_{\alpha, \beta \in E}\left|x_{\beta}^{\prime}\right|\left|x_{\alpha}^{\prime}-x_{\alpha}^{\prime}\right| \cdot \sum_{\lambda \in E}\left|b_{\lambda+\alpha-\beta}\right| \leq\left(1+\frac{\varepsilon}{4}\right) \tau d
\end{aligned}
$$

Finally,

$$
\begin{aligned}
\left\|\Phi(x)-\Phi\left(x^{\prime}\right)\right\| & \leq\left\|\sigma_{1}\right\|+\left\|\sigma_{2}\right\| \leq \frac{\varepsilon}{4} d+\tau\left(1+\frac{\varepsilon}{4}\right) d \\
& =d\left(\tau\left(1+\frac{\varepsilon}{4}\right)+\frac{\varepsilon}{4}\right)=d\left(\theta\left(1-\frac{\varepsilon}{4}\right)+\frac{\varepsilon}{4}\right)=\theta_{1} \cdot d .
\end{aligned}
$$

We can choose $\tau$ to satisfy both inequalities in (ii) and (iii). Indeed put $\tau=\lambda \varepsilon$. Then we need $\frac{1}{5} \leq \lambda \leq 1$ and $\lambda<\lambda(\varepsilon)=\left(1-\frac{\varepsilon}{4}\right) /\left(\varepsilon\left(1+\frac{\varepsilon}{4}\right)\right)$. But $\min _{0<\varepsilon \leq 1} \lambda(\varepsilon)=\lambda(1)=\frac{3}{5}$; therefore, any $\tau, \frac{1}{5} \varepsilon \leq \tau \leq \frac{3}{5} \varepsilon, 0<\varepsilon<1$, is good for both (ii) and (iii).

By the Contractive Mapping Theorem, $\Phi$ has a (unique) fixed point. It proves the main theorem. 
The method used in the proof of the main theorem is applicable to a wide class of (countable) groups. Analogous results hold, for instance, for divisible abelian groups and for direct sums of cyclic groups of prime orders. The complete description of the class of countable (not necessarily abelian) groups admitting the analog of the main theorem is an attractive problem.

Let $U$ be a unitary operator on a Hilbert space $H$. A vector $f \in H$ is called rigid with respect to $U$ if $\lim _{k \rightarrow \infty}\left\|U^{n_{k}} f-f\right\|=0$ for some sequence $\left\{n_{k}\right\}, n_{k} \nearrow \infty$. A unitary operator is mildly mixing if it has no nonzero rigid vectors. A unitary operator $U$ is strongly mixing if $\left\{U^{n} f\right\}$ weakly tends to 0 as $n \rightarrow \infty$ for all $f \in H$. (We use here, for general unitary operators, the terminology which is traditional in the case of operators induced by measurepreserving transformations.) It is known that

strong mixing $\Rightarrow$ mild mixing $\Rightarrow$ weak mixing ( $\equiv$ continuous spectrum).

The classes of mildly mixing and strongly mixing operators can be characterized in terms of properties of sequences along which weak wandering occurs for a dense set of vectors in $H$. Namely, a unitary operator $U$ is mildly mixing if and only if for any $I P$-set $E \subset \mathbb{N}, f \in H$, and $\varepsilon>0$ there exist $\tilde{f} \in H$, $\|f-\tilde{f}\|<\varepsilon$, and an IP-set $\widetilde{E} \subset E$ such that $\left\langle U^{n} \tilde{f}, U^{m} \tilde{f}\right\rangle=0$ for all distinct $n, m \in \widetilde{E}$.

Moreover, $U$ is strong mixing if and only if for any infinite subset $S \subset \mathbb{N}$, $f \in H$, and $\varepsilon>0$ there exist $\tilde{f} \in H,\|f-\tilde{f}\|<\varepsilon$, and an infinite subset $A \subset S$ such that $\left\langle U^{n} \tilde{f}, U^{m} \tilde{f}\right\rangle=0$ for any distinct $n, m \in F S(A)$.

Question. What is the description of the family $P$ of unitary operators with continuous spectrum acting on the Hilbert space $H$ which have the property that for any $f \in H$ and $\varepsilon>0$ there exist $\tilde{f},\|\tilde{f}-f\| \leq \varepsilon$, and a set $S \subset \mathbb{N}$ having positive upper density (i.e., $\left.\lim \sup _{K \rightarrow \infty}[\#(S \cap[1, K]) / K]>0\right)$ such that $\left\langle U^{n} \tilde{f}, U^{m} \tilde{f}\right\rangle=0$ for all distinct $n, m \in S$ ?

The following remark shows that there exist unitary operators with continuous spectrum which do not belong to $P$. Let us call a unitary operator rigid if every $f \in H$ is rigid. There exist rigid operators with continuous spectrum, and it can be shown that for such operators no nonzero vector can wander along a sequence of positive upper density.

Let $T$ be a measure-preserving transformation of a probability space $(\Omega, \mu)$, and let $U$ be the unitary operator on $L^{2}(\Omega, \mu)$ induced by $T$. In this special case instead of vectors in $L^{2}(\Omega, \mu)$, one can consider measurable partitions of $\Omega$ into a finite number of sets. It is shown in $[\mathrm{K}]$ that $T$ is weakly mixing if and only if weakly independent partitions (i.e., partitions $\xi$ for which there exists an infinite sequence $\left\{n_{i}\right\}_{i=1}^{\infty}$ such that $\left\{T^{n_{i}} \xi\right\}$ are mutually independent) are dense in the space of all partitions. We do not know if the method of this note is applicable to the case of partitions.

\section{REFERENCES}

[F] H. Furstenberg, Recurrence in ergodic theory and combinatorial number theory, Princeton Univ. Press, Princeton, NJ, 1981.

[G] V. Graham, Weakly wandering vectors for compactly generated groups of unitary operators, J. Math. Anal. Appl. 46 (1974), 565-594. 
[K] U. Krengel, Weakly wandering vectors and weakly independent partitions, Trans. Amer. Math. Soc. 164 (1972), 199-226.

[KN] B. Koopman and J. von Neumann, Dynamical systems of continuous spectra, Proc. Nat. Acad. Sci. U.S.A. 18 (1931), 255-263.

(V. Bergelson and B. Mityagin) Department of Mathematics, The Ohio State University, Columbus, Оніо 43210-1174

(V. Bergelson) Department of Mathematics, Technion-IsRael Institute of Technology, 32000 HAIFA, ISRAEL

(I. Kornfeld) Department of Mathematics, North Dakota State University, Fargo, NORTH DAKOTA 58105 